\title{
Microbiology Specimen Examination Baseline Flag
}

National Cancer Institute

\section{Source}

National Cancer Institute. Microbiology Specimen Examination Baseline Flag. NCI

Thesaurus. Code C87906.

An indication or description of a baseline value within a microbiology specimen

examination. 